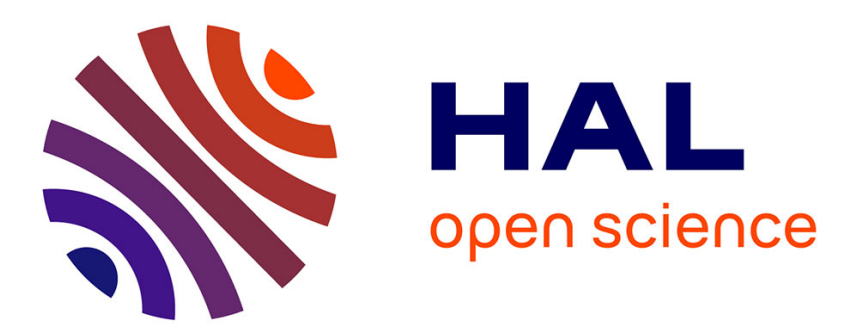

\title{
FreeSplit: A Write-Ahead Protocol to Improve Latency in Distributed Prefix Tree Indexing Structures
}

\author{
Rudyar Cortés, Xavier Bonnaire, Olivier Marin, Pierre Sens
}

\section{To cite this version:}

Rudyar Cortés, Xavier Bonnaire, Olivier Marin, Pierre Sens. FreeSplit: A Write-Ahead Protocol to Improve Latency in Distributed Prefix Tree Indexing Structures. [Research Report] RR-8637, INRIA Paris. 2014. hal-01092251

\section{HAL Id: hal-01092251 \\ https://hal.inria.fr/hal-01092251}

Submitted on 8 Dec 2014

HAL is a multi-disciplinary open access archive for the deposit and dissemination of scientific research documents, whether they are published or not. The documents may come from teaching and research institutions in France or abroad, or from public or private research centers.
L'archive ouverte pluridisciplinaire HAL, est destinée au dépôt et à la diffusion de documents scientifiques de niveau recherche, publiés ou non, émanant des établissements d'enseignement et de recherche français ou étrangers, des laboratoires publics ou privés. 
FreeSplit: A

Write-Ahead Protocol to

Improve Latency in

Distributed Prefix Tree

Indexing Structures

Rudyar Cortés, Xavier Bonnaire, Olivier Marin, Pierre Sens

RESEARCH

REPORT

$\mathbf{N}^{\circ} 8637$

November 2014

Project-Teams ARMADA 



\title{
Iñ̄iá
}

\section{FreeSplit: A Write-Ahead Protocol to Improve Latency in Distributed Prefix Tree Indexing Structures}

\author{
Rudyar Cortés, Xavier Bonnaire, Olivier Marin, Pierre Sens \\ Project-Teams ARMADA \\ Research Report $\mathrm{n}^{\circ} 8637$ - November 2014 - 16 pages
}

\begin{abstract}
Distributed Prefix Tree indexing structures on top of peer-to-peer overlays provide a scalable solution to support range queries and proximity queries for Big Data applications. However, the latency of current maintenance protocols impacts very negatively on main operations like data insertions. This paper presents a new maintenance protocol that anticipates every data insertion on provisional child nodes. A performance evaluation conducted on the Prefix Hash Tree and FreeSplit shows that FreeSplit significantly reduces maintenance overheads, and therefore improves query response time.
\end{abstract}

Key-words: Trie indexing, Big Data, Maintenance Protocol

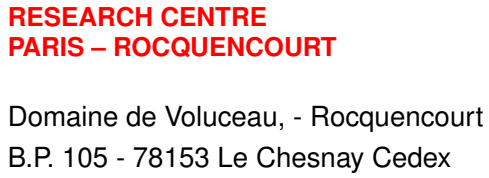




\section{FreeSplit: A Write-Ahead Protocol to Improve Latency in Distributed Prefix Tree Indexing Structures}

Résumé : Pas de résumé

Mots-clés : calcul formel, base de formules, protocole, différentiation automatique, génération de code, modélisation, lien symbolique/numérique, matrice structurée, résolution de systèmes polynomiaux 


\section{Contents}

\begin{tabular}{lll}
\hline 1 & Introduction & 3 \\
\hline
\end{tabular}

2 Dynamic Trie-Based Indexing Structures

\begin{tabular}{llr}
\hline 3 & FreeSplit: a Write-Ahead Protocol & 6
\end{tabular}

4 Evaluation $\quad 9$

4.1 Split time . . . . . . . . . . . . . . . . . . . . 10

4.1 .1 Impact of the insertion rate . . . . . . . . . . . . . . . . . . . . 11

4.1.2 Impact of the skewness . . . . . . . . . . . . . . . . . . . . 11

4.2 Insertion Failure $\ldots \ldots \ldots \ldots$. . . . . . . . . . . . . . . . . . . . 12

4.2 .1 Impact of the insertion rate . . . . . . . . . . . . . . . . . . . . . 12

4.2 .2 Impact of the skewness . . . . . . . . . . . . . . . . . . . . . . . . . . . . . . . . . . . . . . .

4.3 Storage cost . . . . . . . . . . . . . . . . . . . 13

4.4 Split message complexity . . . . . . . . . . . . . . . . . . . . . . . 13

4.5 Insertion Cost . . . . . . . . . . . . . . . . . . . . . . . . . . . . . . 13

\begin{tabular}{|lr|}
5 Discussion & 14 \\
\hline
\end{tabular}

5.1 Message Cost . . . . . . . . . . . . . . . . . . . . . . 14

5.2 Insertion rate $(\mathrm{R})$ versus storage capacity $(\mathrm{B})$. . . . . . . . . . . . . . . . . . . . . . . . . . . . . . . . .

5.3 Storage space overhead . . . . . . . . . . . . . . . . . . . . . . 14

5.4 High Availability . . . . . . . . . . . . . . . . . . . . . . . . . . . . . . . . . . . . . . . . . . . . .

5.5 Portability . . . . . . . . . . . . . . . . . . . . . . 14

\begin{tabular}{llr}
\hline 6 & Related Work & 15
\end{tabular}

\begin{tabular}{llr}
\hline 7 Conclusion & 15 \\
\hline
\end{tabular}

\section{Introduction}

Indexing Big Data is a major issue towards extracting key information from applications which generate massive and dynamic inputs of small objects coming from millions of users on a daily basis [1 [2] 3. Big Data applications, like digital health and personal sports training [4] [5] or sensing as a service [6] [7, generate high loads of insertions without deletions. Tree-based dynamic indexing over structured peer-to-peer overlays like Prefix Hash Tree (PHT) [8, LIGHT [9], Distributed Segment Tree (DST) [10, or Range Search Tree (RST) [1] supports indexing and range queries on a large scale. These solutions distribute object keys efficiently by using a decentralized tree-based indexing scheme mapped onto a Distributed Hash Table like Pastry [12] or Chord 13 .

Distributed Prefix Tree indexing structures rely on a maintenance protocol that dynamically distributes data on new trie nodes through high-latency internet links based on data distribution. Achieving the lowest latency in the maintenance of distributed trie structures is a key task as it allows to minimise the side effect of maintenance on insertions. Leaf nodes performing the maintenance protocol delay or even discard insertions, thus increasing query response times. Concurrent and continuous inputs on a large scale produce a heavy load on trie nodes: the dynamic configuration of new trie nodes also increases query response times. Therefore, massive incoming flows in trie-based indexing systems significantly deteriorate overall system performance because of the high maintenance cost they induce. 
In this paper we present a new approach, called FreeSplit, which reduces the impact of split operations, thus improving the response time for data insertions. FreeSplit anticipates maintenance operations by performing every insertion on the target node and on provisional child nodes. This drastically improves the performance of prefix trees when indexing massive input flows of small objects.

The main contributions of this paper are: (a) a study of the cost of maintenance operations on a trie-based indexing system like PHT, and (b) a new maintenance operation called FreeSplit which drastically reduces the index maintenance cost on distributed prefix tree structures.

Section 2 gives an overview of structures like the Prefix Hash Tree. Section 3 explains the new mechanisms introduced by FreeSplit, and how to implement our solution on top of PHT. Section 4 provides a detailed performance evaluation of FreeSplit. The discussion in section 5 shows the benefits of using FreeSplit in the context presented at the beginning of this introduction: massive insertions due to voluminous and continuous incoming flows of small objects. In this context, FreeSplit significantly outperforms PHT. Section 6 presents a brief state of the art of tree-based indexing structures, and section 7 concludes and draws perspectives.

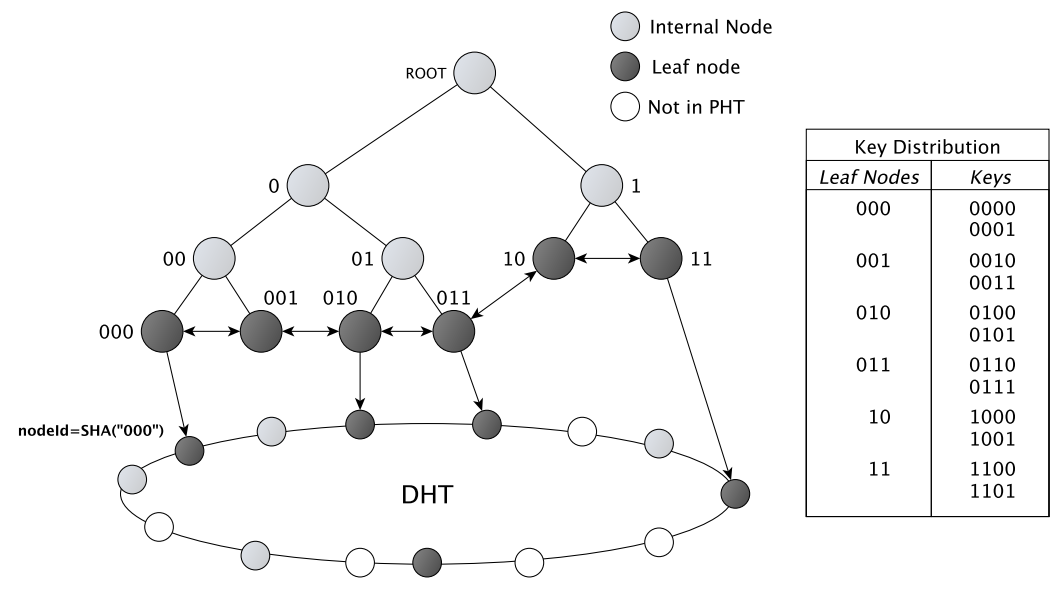

Figure 1: PHT Structure

\section{Dynamic Trie-Based Indexing Structures}

Prefix trees, such as the Prefix Hash Tree (PHT) 8] and LIGHT [9, allow distributed indexing by means of recursive space partitioning. The trie dynamically evolves upon index updates: an overloaded leaf node splits the data it stores onto two new child nodes following a defined space partitioning rule.

This paper presents an enhancement that improves any trie-based indexing structure. For the sake of clarity, we will explain our solution using the Prefix Hash Tree (PHT).

PHT is a distributed indexing data structure built over a DHT [12] [13]. This structure creates data locality by using a prefix rule to map trie nodes over a DHT ring. This allows to support complex queries such as range queries, proximity queries and MIN/MAX queries.

PHT comprises three node states: leaf nodes store data, internal nodes maintain the trie structure, and external nodes belong to the DHT ring but not to the trie. 


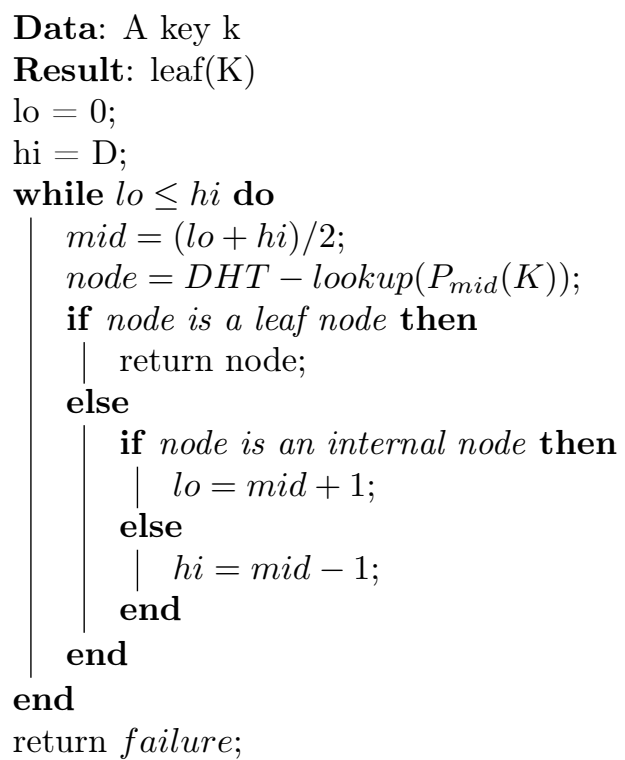

Algorithm 1: PHT-LOOKUP-BINARY

PHT associates a recursively defined label with every node. The left child node inherits the label of its parent node concatenated with 0 , and the right child node with 1 . Data storage follows the same prefix rule: looking up an object with key $k$ consists in finding a leaf node which label is a prefix of $k$. This strategy recursively divides the domain space $\{0,1\}^{D}$, where $D$ is the amount of bits used to represent keys, and delivers an implicit knowledge about the location of every object in the system. This strategy recursively divides the domain space $\{0,1\}^{D}$, where $D$ is the amount of bits used to represent keys, and delivers an implicit knowledge about the location of every object in the system. PHT also maintains a doubly-linked list of all leaf nodes in order to provide sequential access.

The logical map between PHT nodes and DHT nodes is generated by computing the Secure Hash Function SHA-1 over the PHT node label. Thus, the DHT nodeId associated to a given PHT node labeled $l$ is nodeId $=S H A(l)$. Figure 1 shows an example of a PHT that indexes the data domain $\{0,1\}^{4}$ and its respective DHT overlay.

When an overloaded leaf node $X$ reaches its maximum storage capacity $B+1$, it carries out a split operation. The overloaded node configures two new child nodes and distributes $B$ objects following the prefix rule. In order to stop data insertions the split node changes its state from leaf node to internal node, and relies on the DHT to route a split message to the nodes that will become its children according to the prefix rule. Every split message is sent in $O(\log (N))$ hops, where $N$ is the DHT size. The split message contains the number of keys to be transferred before the child can change its state from external to leaf, and the IP address of the neighbour node that is relevant to the recipient: left neighbor for the left-hand child and right neighbor for the right-hand child.

Upon reception of the split message, the left-hand child node returns its IP address to its new parent node and sends a message to its new sibling node in order to update the doubly-linked list of leaf nodes. Note that the update message to the right-hand sibling is routed via the DHT. The right-hand child does the same, but waits for the message of its left sibling to avoid DHT routing. Once it has acquired the IP address of a child, the split node transfers all the local data blocks that match the label of the child. When this transfer is over, the child becomes a leaf 
node.

Algorithm 1 presents the binary lookup algorithm used by PHT [8 for insertions. This algorithm takes key $k$ as input and returns the leaf node with the longest prefix of $k$ as label by sending $O(\log (D))$ node state requests routed via the DHT ring, with $D$ the number of bits used to represent keys. While an internal node with label $l$ is configuring two new leaf nodes, there are no available leaf nodes associated to $l 0$ and $l 1$, and therefore all lookups over a key with prefix $l$ will fail until the split operation finishes and child nodes become available to process requests. In this case, the client node must wait some time $\Delta t$ before retrying the insertion. This increases both the response time and the overall message overhead in the network.

A range query consists in contacting all leaf nodes whose label fits within the given data range. A Sequential range query over a data interval $I=[L, M]$ starts by locating the leaf node that indexes the lower bound $L$ and crawls through the doubly-linked list until it reaches the leaf node that indexes the upper bound $M$. The crawling of the doubly-linked list can be avoided by performing a parallel search. A parallel range query starts by locating the node whose label shares the largest common prefix with the lower and the upper bound. This node recursively forwards the query until all leaf nodes that cover the range are reached.

\section{FreeSplit: a Write-Ahead Protocol}

This section details FreeSplit, our write-ahead protocol for distributed prefix tree structures. The goal of FreeSplit is to decrease the latency of split operations by using a write-ahead technique on provisional nodes upon each insertion, regardless of the split condition. Thus when the split condition occurs, the latency of the split operation is reduced to the transfer of a single object from the parent node to one of its children.

Our write-ahead protocol follows the recursive space partitioning rule and introduces a new node state called next leaf. Let A be a client node that indexes a given data item with key $k$, and let $X$ be the leaf node whose label $l$ is a prefix of $k$. The insertion of $k$ on $X$ is written-ahead to a provisional child node whose label starts with $l$ and shares one additional bit with $k$.

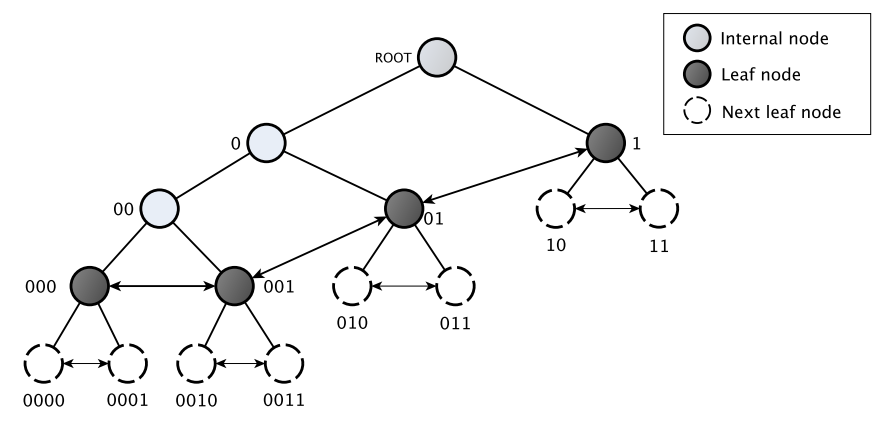

Figure 2: Representation of the write-ahead protocol on top of the PHT structure.

Figure 2 represents the structure produced by our protocol on top of PHT. A leaf node with label $l$ is parent to two next leaf nodes: the left one corresponds to label $l 0$ while the right one corresponds to $l 1$. A next leaf node stores the static and dynamic reference to its direct sibling. For example, the next leaf node labeled $l=010$ stores the reference to its direct sibling node labeled $l=011$ and vice versa. 
At start-up all DHT nodes are external, they do not belong to the trie. The initialization of the FreeSplit trie consists in setting up its foundation: the root node and all the children associated with a single digit label (these constitute the leaf nodes at startup), as well as all next leaf nodes.

Algorithm 2 shows the pseudocode of the FreeSplit init routine on top of PHT.

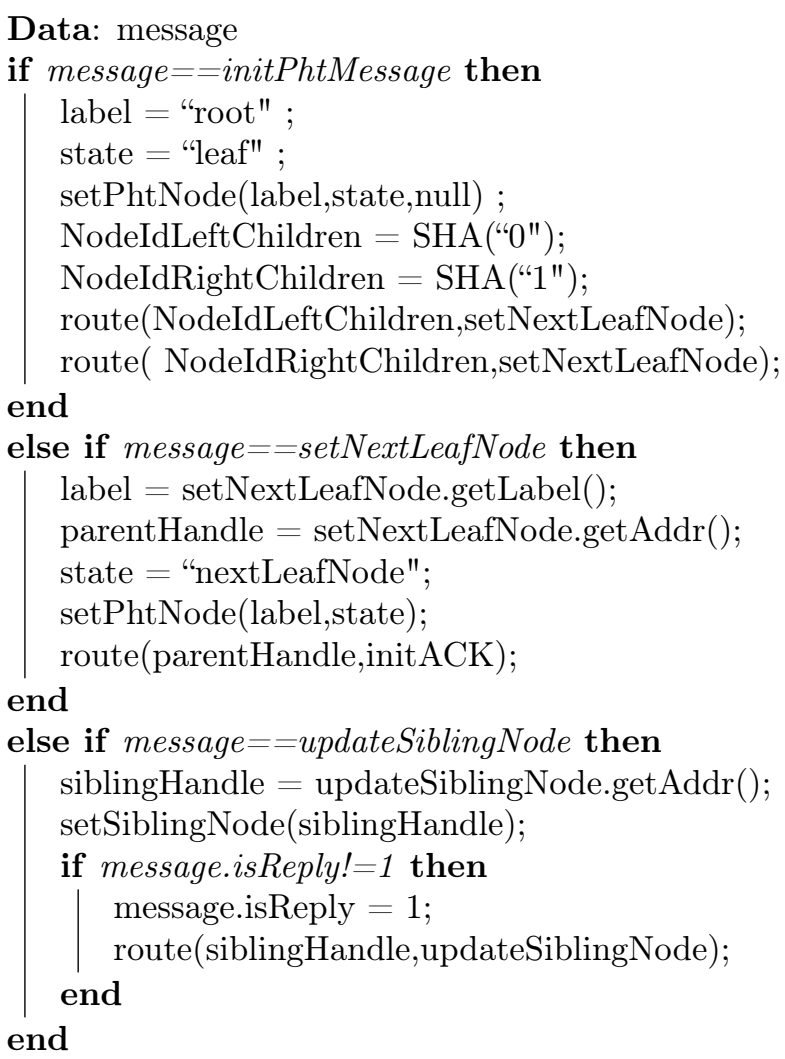

Algorithm 2: Write-Ahead PHT initialization

The first step of the trie initialization is to route a initPhtMessage to the nodeId associated with the label of the root node: NodeId $d_{\text {root }}=S H A$ ("root"). When the root node receives the initPhtMessage it routes a setNextLeafNode message to both its child nodes labeled $l_{0}=0$ and $l_{1}=1$. The setNextLeafNode message contains the label associated to the node and all references to sibling nodes. Note that at startup all these references are null. Every next leaf node initializes its data structure and returns an init $A C K$ message to the root node with its IP address. Next leaf nodes also initialize partially their double linked list by exchanging an updateSiblingNode message with their direct sibling. For instance, the left leaf node labeled 10 routes its updateSiblingNode message to the right sibling node labeled $l 1$. Upon reception the right sibling acquires the IP address of its left sibling and responds directly with its own updateSiblingNode message. The initialization process ends when the root node receives an initACK message from both its child nodes and registers their IP address.

This strategy anticipates the split operation and reduces its cost in terms of latency. Upon reaching the split condition, the overloaded node changes its state from leaf to internal and transfers the single latest object to the corresponding next leaf node. The splitting node then sends a split message directly to the IP address of its next leaf nodes to notify them that a split 
is under way. The reception of a split message on a next leaf node triggers a state update: the recipient becomes a leaf node. It then updates its doubly-linked list with the IP addresses sent in the split message, and routes a setNextLeafNode message to initialize its own next leaf nodes according to the prefix rule.

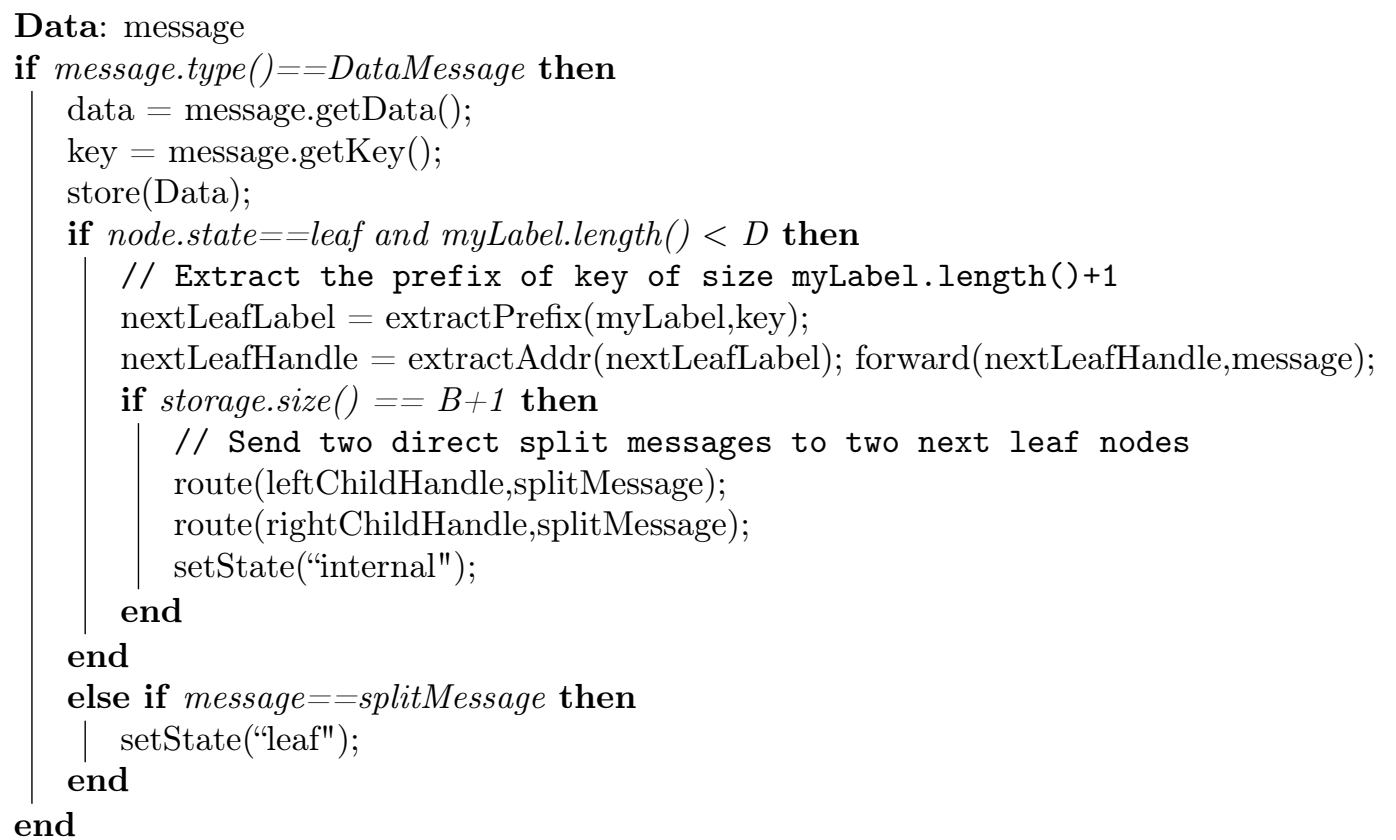

Algorithm 3: Write-Ahead indexing

Given the write-ahead objective of the FreeSplit structure, indexing a given data item with key $k$ actually involves two data insertions. Algorithm 3 gives the pseudocode of the FreeSplit insertion operation. The client carries out the first insertion on the leaf node $L_{N}$ whose label is a prefix of $k$, and in turn $L_{N}$ carries out the second insertion onto its next leaf node whose label is also a prefix of $k$.

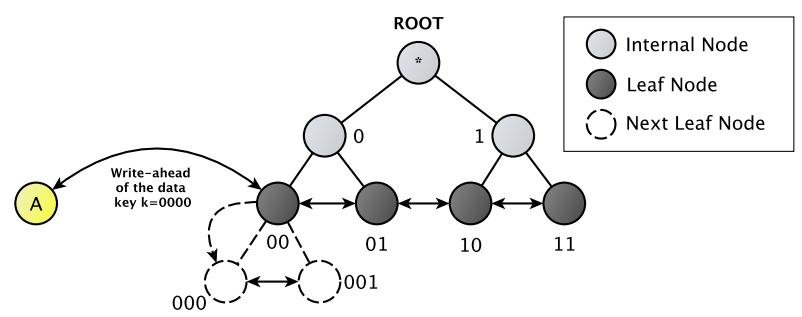

Figure 3: Write-ahead protocol example: insertion of data key $\mathrm{k}=0000$.

Figure 3 shows an example of the insertion operation. Client node $A$ inserts an object with key $k=0000$. First, $A$ indexes $k$ onto the leaf node labeled $l=00$. Then, the leaf node copies $k$ onto its next leaf node labeled $l_{1}=000$.

Figure 4 presents an example of a FreeSplit split on top of PHT. The node labeled $l=00$ 
changes its state from leaf to internal and sends a split message to both its next leaf nodes (labeled $l=000$ and $l=001$ ). In turn, these nodes change their state from next leaf to leaf and send a setNextLeafNode message to their respective next leaf nodes.

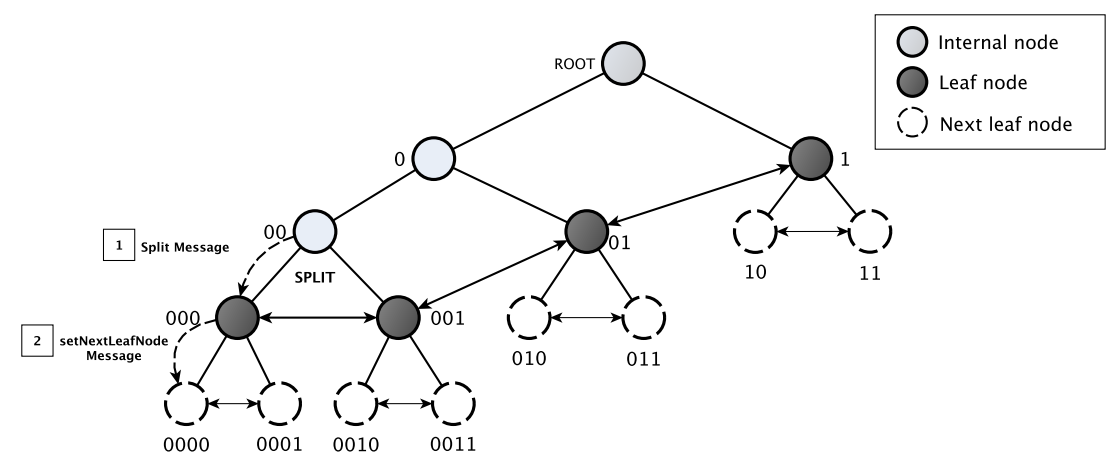

Figure 4: Write ahead protocol example. Node labeled $l=00$ performs a split operation

\begin{tabular}{|l|l|}
\hline Parameter & Value \\
\hline Number of nodes (N) & 100 \\
\hline Storage capacity (B) & 250 keys \\
\hline Keys length (D) & 32 bits \\
\hline
\end{tabular}

Table 1: Trie configuration parameters

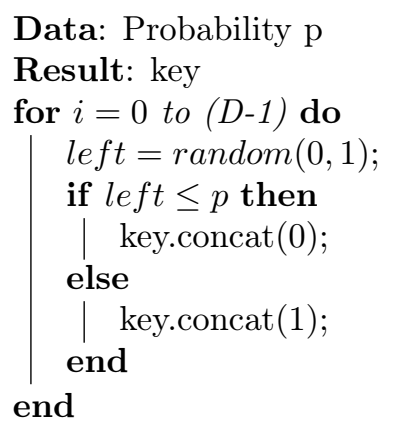

Algorithm 4: Keys generation algorithm

\section{Evaluation}

In this section we present experimental results obtained by deploying PHT and FreeSplit on top of FreePastry, an open-source implementation of Pastry [12. We compare our write-ahead protocol with PHT by assessing their performance in terms of split and lookup operations, as well as in terms of their storage cost and message overhead. 


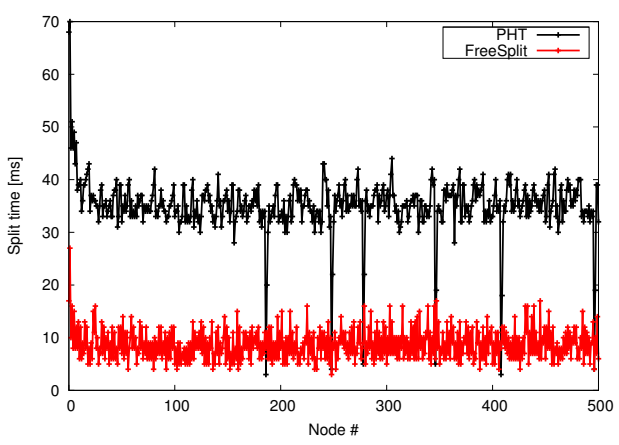

(a) $\mathrm{R}=10$ keys $/$ second

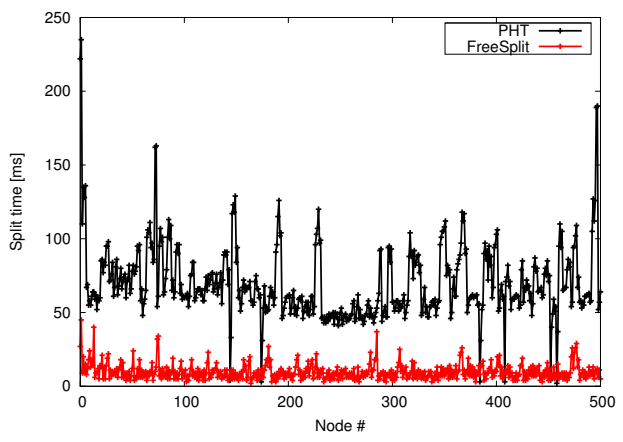

(c) $\mathrm{R}=250$ keys $/$ second

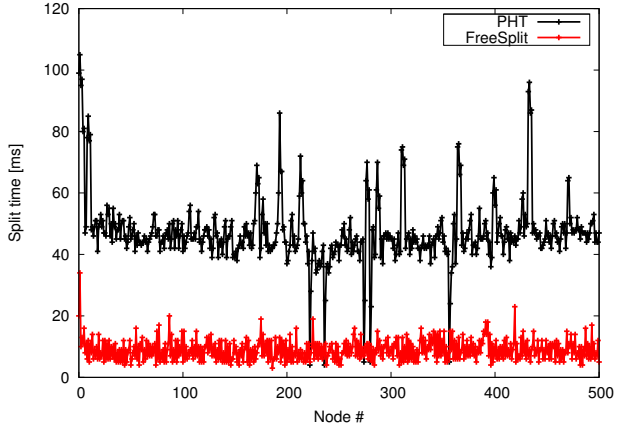

(b) $\mathrm{R}=100 \mathrm{keys} /$ second

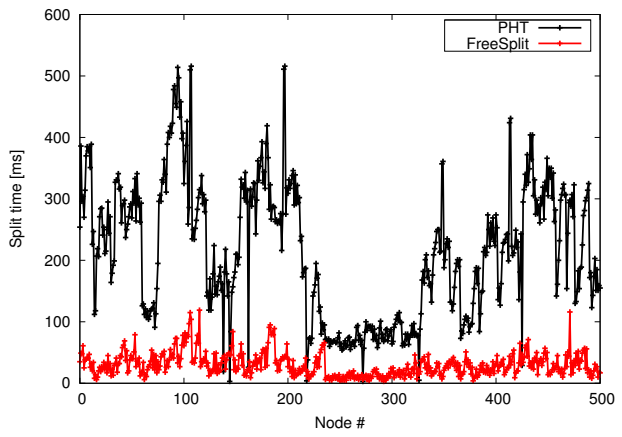

(d) $\mathrm{R}=500$ keys/second

Figure 5: Split time when indexing a uniform distribution for different insertion rates

All experiments reported in this paper were run on a single Java VM version 1.6.0-65 using an intel core i7 $2.6 \mathrm{Ghz}$ with $8 \mathrm{~GB}$ of main memory and OS X 10.9.1. Table 1 sums up the configuration parameters for PHT and FreeSplit. A leaf node performs a split operation when the number of objects stored is equal to $B=250$ objects.

We measured the impact of the insertion rate and of the key distribution on the performance of the split operation. In every experiment, one single node is chosen randomly in order to index 50,000 keys at insertion rates of $\mathrm{R}=10,100,250$, and 500 keys/second. We generated three key distributions with different levels of skewness following algorithm 4

- Uniform distribution $(\mathbf{p}=\mathbf{0 . 5})$. Distributes roughly $0.5 B$ keys to each child node at every split operation.

- Skewed distribution $(\mathbf{p}=\mathbf{0 . 3})$. Distributes roughly $0.3 B$ keys to the left child node and $0.7 B$ keys to the right child node.

- Very skew distribution $(\mathbf{p}=\mathbf{0 . 1})$. Distributes roughly $0.1 B$ keys to the left child node and $0.9 B$ to the right child node.

\subsection{Split time}

In this subsection we compare PHT and FreeSplit by assessing the performance of their split operations. For this purpose, we define the split time as the time elapsed from the instant the 


\begin{tabular}{|c|c|c|c|c|c|c|c|c|c|}
\hline \multicolumn{3}{|c|}{ Very Skewed $(p=0.1)$} & \multicolumn{2}{|c|}{ Skewed $(p=0.3)$} & \multicolumn{2}{|c|}{ Uniform $(\mathrm{p}=0.5)$} & \multirow[b]{2}{*}{$\begin{array}{l}\text { PHT } \\
\text { Average }\end{array}$} & \multirow[b]{2}{*}{$\begin{array}{l}\text { FS } \\
\text { Average }\end{array}$} & \multirow[b]{2}{*}{$\begin{array}{l}\text { Average } \\
\text { Speedup }\end{array}$} \\
\hline $\begin{array}{l}\text { Rate } \\
\text { keys/s }\end{array}$ & $\begin{array}{l}\text { PHT } \\
{[\mathrm{ms}]}\end{array}$ & $\begin{array}{l}\text { FS } \\
{[\mathrm{ms}]}\end{array}$ & $\begin{array}{l}\text { PHT } \\
{[\mathrm{ms}]}\end{array}$ & $\begin{array}{l}\text { FS } \\
{[\mathrm{ms}]}\end{array}$ & $\begin{array}{l}\text { PHT } \\
{[\mathrm{ms}]}\end{array}$ & $\begin{array}{l}\text { FS } \\
{[\mathrm{ms}]}\end{array}$ & & & \\
\hline 10 & 25.49 & 8.22 & 30.41 & 8.59 & 35.18 & 8.78 & 30.36 & 8.53 & 3.55 \\
\hline 100 & 34.07 & 8.28 & 40.32 & 8.62 & 45.86 & 8.83 & 40.08 & 8.57 & 4.67 \\
\hline 250 & 50.59 & 8.72 & 56.35 & 8.78 & 70.48 & 9.84 & 59.14 & 9.11 & 6.48 \\
\hline 500 & 163.11 & 26.10 & 139.39 & 16.10 & 221.32 & 31.47 & 174.60 & 24.55 & 7.11 \\
\hline
\end{tabular}

Table 2: Average split time when a node indexes 50,000 keys

split condition is reached until the instant both new child leaf nodes become available for lookups. We measure the impact of the insertion rate and data distribution on this metric.

\subsubsection{Impact of the insertion rate}

Table 2 shows the average split time of PHT and FreeSplit (FS) with respect to the data distribution and insertion rate. When the insertion rate is $\mathrm{R}=10 \mathrm{keys} / \mathrm{second}$ FreeSplit presents an average split time 3.55 times faster than PHT ( $8.53 \mathrm{~ms}$ and $30.36 \mathrm{~ms}$ respectively). The split time of FreeSplit represents the minimal time required to configure two new child nodes in advance.

Up to the point where the insertion rate reaches the storage capacity of a single leaf node $(\mathrm{R}=\mathrm{B}=250)$, FreeSplit exhibits a near constant split time. In comparison, the split time increases very fast in PHT. At insertion rates greater than the storage capacity of a single node, FreeSplit presents a split time 7.1 times faster than PHT. However, at this insertion rate leaf nodes have less time to configure next leaf nodes in advance.

A high rate of insertions produces a high load of lookup requests which have an impact on the split time. Figure 5 presents the split time measured by every node in a single test for different insertion rates. As the insertion rate increases, lookups impact ever more on the split time. This phenomenon appears clearly in figure 5 when an uniform data distribution is indexed at different insertion rates from $\mathrm{R}=10$ to $\mathrm{R}=500$ keys/second. We chose to show this distribution because it illustrates well the effect of FreeSplit on the split time as nodes get roughly the same number of keys, and therefore must measure similar split times.

Figure $5 \mathrm{a}$ shows the split time measured for trie nodes with a low insertion load. In this case, FreeSplit decreases the split time at every split operation. Nodes at the highest trie levels present the highest times because their insertion load is greater than nodes at the lowest levels. When the insertion load increases to $\mathrm{R}=500 \mathrm{keys} /$ second as in figure $5 \mathrm{~d}$ trie nodes become saturated with lookup requests: this induces unstable split times. FreeSplit reduces the impact of this load on the split time because the write-ahead protocol allows nodes to copy data before nodes become overloaded.

\subsubsection{Impact of the skewness}

In order to assess the impact of the skewness on the split time we indexed three key distributions: from very skewed to uniform. Table 2 presents the average split time of PHT and FreeSplit for these distributions. Note that, conversely to the insertion rate, key distribution skewness does not affect the split time of FreeSplit. Such is not the case for PHT, which performs better with a uniform distribution and yet always performs worse than FreeSplit whatever the distribution. 


\begin{tabular}{|c|c|c|c|c|c|c|}
\hline \multicolumn{3}{|c|}{ Very Skewed $(p=0.1)$} & \multicolumn{2}{|c|}{ Skewed $(p=0.3)$} & \multicolumn{2}{|c|}{ Uniform $(p=0.5)$} \\
\hline $\begin{array}{l}\text { Rate } \\
\text { keys/s }\end{array}$ & PHT & $\mathrm{FS}$ & $\mathrm{PHT}$ & FS & $\mathrm{PHT}$ & $\mathrm{FS}$ \\
\hline 10 & 0 & 0 & 0 & 0 & 0 & 0 \\
\hline 100 & 88 & 0 & 39 & 0 & 0 & 0 \\
\hline 200 & 240 & 5 & 113 & 2 & 98 & 3 \\
\hline 250 & 363 & 60 & 190 & 39 & 151 & 28 \\
\hline 500 & 2586 & 1125 & 1138 & 690 & 1166 & 543 \\
\hline
\end{tabular}

Table 3: Average number of insertion fails when a node indexes 50,000 keys

\subsection{Insertion Failure}

In this subsection we analyse the impact of the split time on the insert operation. An insertion over a key $k$ fails when the lookup algorithm is not able to reach an available leaf node with a label that is a prefix of $k$. This happens when a node is splitting into two new leaf nodes: there is then no node available to process requests (see section 2 for further details). We measured the number of insertion failures when a single node indexes 50,000 keys with no retries. Table 3 shows the average number of insertion failures with respect to the insertion rate for different key distributions. FreeSplit overcomes PHT by reducing the number of insertion failures in all cases.

\subsubsection{Impact of the insertion rate}

When the insertion rate increases, the average number of insertion failures increases as a direct consequence of the increasing split time. When the insertion rate is lower than the storage capacity of a single node $(R<B)$ the number of insertion failures presented by FreeSplit is significantly lower than PHT. For instance, when the insertion load is lower than $\mathrm{R}=100$ keys/second FreeSplit does not incur any insertion failure. Increasing the insertion rate to $\mathrm{R}=200$ keys/second produces a maximum number of 5 insertion failures for FreeSplit, to be compared with 240 for PHT.

When the insertion rate increases until $R \geq B$, the number of insertion failures increases. An insertion rate higher than the storage capacity of trie nodes produces a high split frequency in the whole trie that increases the number of insertion failures. In this scenario, FreeSplit still incurs less insertion failures than PHT. For instance, for $\mathrm{R}=250$ keys/second FreeSplit incurs 5.39 times less insertion failures when the data distribution is uniform and 6.09 times less when the data distribution is very skewed.

\subsubsection{Impact of the skewness}

Results presented in table 3 show that the number of insertion failures increases with the data skewness. The more skewed the data distribution, the more insertions are concentrated in a single trie branch. And yet, FreeSplit still incurs less insertion failures than PHT for different levels of data skewness.

When the insertion rate is lower than the storage capacity of a single node $(R<B)$ the impact of the data skewness is minimal for FreeSplit. For instance as mentioned before, FreeSplit incurs 5 insertion failures while PHT incurs 240 when the insertion rate is $\mathrm{R}=200$ keys/second and the data distribution is very skewed. When $R \geq B$, the number of insertion failures increases in both cases. In these cases, FreeSplit overcomes PHT, but its performance decreases. For 
instance when a very skewed distribution is indexed, FreeSplit incurs 6.05 times less insertion failures than PHT for $\mathrm{R}=250$, and 2.29 times less when $\mathrm{R}$ increases to $500 \mathrm{keys} / \mathrm{second}$.

\subsection{Storage cost}

FreeSplit replicates every data item twice (one in the current leaf node and another one in the next leaf node). Let $n_{k}$ the number of keys indexed in a given PHT. The storage cost incurred by FreeSplit is given by.

$$
C_{\text {Storage-FreeSplit }}=2 \times n_{k}=2 \times C_{\text {Storage }-P H T}
$$

\subsection{Split message complexity}

In PHT, a leaf node reaches its split condition when it receives an insertion request and already stores $B$ objects. The total cost combines the node coordination cost and the object transfer cost.

Equation 2 computes the message complexity of the node coordination for a split operation in PHT: it sums up the total cost incurred by routing the split messages and adds the update cost of the leaf links.

$$
C_{\text {coordination }}=3 \times(O(\log (N))+1)
$$

The object transfer cost represents the number of messages required to dispatch $B+1$ objects from the parent node to its children. Since we assume that each object is small enough to be transferred using a single TCP message, the message complexity of the object transfer equals the maximum number $B$ of objects that a node can store.

Therefore, equation 3 computes the total message complexity of a split operation in PHT.

$$
C_{P H T-S p l i t}=3 \times(O(\log (N))+1)+(B+1)
$$

Compared to PHT, FreeSplit spend only two extra direct split messages to the IP address of its next leaf nodes to notify them that the split operation is under way. Therefore, the number of messages incurred by FreeSplit in a split operation is given by equation 4.

$$
C_{\text {FreeSplit }}=C_{P H T-S p l i t}+2 .
$$

\subsection{Insertion Cost}

A single data insertion generates $O(\log (D))$ DHT routing messages. Thus, the number of messages generated by a single insertion is given by equation 5 .

$$
C_{\text {insertion }}=O(\log (D)) \times O(\log (N))
$$

An insertion that generates a lookup failure must retry $n$ times. Thus, the number of messages generated by $n$ insertion tries is given by equation 6 .

$$
C_{\text {insertion }}=(n+1) \times(O(\log (D)) \times O(\log (N)))
$$

FreeSplit drastically reduces the number of insertion failures (see table 3) and therefore the number of messages generated by insertions. 


\section{Discussion}

\section{$5.1 \quad$ Message Cost}

FreeSplit only sends two extra direct IP messages compared to PHT in order to notify to the next leaf nodes that a new split operation is under way (see equation 4). Therefore, FreeSplit exhibits the same scalability as PHT.

\subsection{Insertion rate $(\mathrm{R})$ versus storage capacity $(\mathrm{B})$}

FreeSplit exhibits the best performance when the insertion rate $\mathrm{R}$ is less or equal to the storage capacity of a single node B. FreeSplit, then copies the maximum number of objects on next leaf nodes while maintaining a minimal split time. Another benefit of FreeSplit compared to PHT is that, if we know the maximum objects insertion rate for a given application, we can choose the appropriate number of objects $B$ per leaf node in order to maintain a minimal split time. This allows a proper setup of a tree-based indexing system for a specific application with massive incoming flows of data.

\subsection{Storage space overhead}

FreeSplit increases the storage space overhead by a factor of 2 (see equation 1). For applications that need to optimise the storage space, the write-ahead protocol can be initiated at a later point (i.e, when a leaf node is $\alpha \%$ of its storage capacity, where $\alpha$ is a system parameter). The value of $\alpha$ must be chosen as a commitment between the maximum insertion rate $\mathrm{R}$ and the storage capacity B as we discussed above. This criteria allows to reduce the storage space overhead below factor 2 .

\subsection{High Availability}

Like PHT, FreeSplit can use replication at the DHT level in order to increase the availability of indexed objects. Replicas are usually stored on numerically close nodes in the DHT to ensure that the overlay can resist to network partitions (in the leafset for Patry or in the $L$ successors for Chord). The same write-ahead operations are carried out within all the replicas of the leaf nodes. Managing replicas induces a higher cost for the maintenance operations. Nevertheless, transferring objects to the replicas of the provisional nodes (next leaves) can also be made in parallel using the same idea of the write-ahead technique. This makes the FreeSplit replication faster than that of PHT, where objects must be replicated at the time of the transfer of the $B+1$ objects to the new leaves upon a split. In FreeSplit, when the next leaves become new leaves, most or all of the replicas of the objects already exist.

\subsection{Portability}

Any type of dynamic tree-based indexing structure that performs a split operation following a defined domain or data partitioning rule can be improved by implementing our write-ahead approach. For example, LIGHT [9] defines an index maintenance operation named incremental leaf split similar to that of PHT. Thus, our write-ahead approach can be implemented in LIGHT in order to anticipate object insertions onto next leaves. 


\section{Related Work}

The literature comprises many solutions to support distributed indexing and range queries on a large scale over Distributed Hash Tables [12] [13]. We classify them into two main groups: static tree-like indexing structures [10 [11] create a static binary tree with $n$ levels by recursive range partitioning, and dynamic trie-like indexing structures [8] 9] dynamically divide the domain space.

Static tree indexing solutions suffer from a lack of load balancing and from significant message overheads because they use the higher levels of the tree (internal nodes) to perform insertions and range queries. The Distributed Segment Tree (DST) [10] and the Range Search Tree (RST) [11] are examples of such indexing structures .

The Distributed Segment Tree (DST) is a tree-like structure that indexes the data domain $R=[A, B]$ of size $n$ by dividing it recursively into subintervals of equal size, creating a static segment tree of $L+1=\lceil(\log (n)+1)\rceil$ levels. In this structure all data is replicated onto internal nodes and requires no index maintenance operations. However, this strategy quickly induces internal nodes saturation and high tree levels can easily overload and become bottlenecks.

The Range Search Tree (RST) is an extension of DST. RST proposes a load balancing mechanism based on a Load Balance Matrix (LBM) and a dynamic band that represents an available tree zone to manage insertions and range queries. LBM performs data partitioning by distributing data onto several nodes in order to avoid node saturation, and the dynamic band distributes the query load according to statistics on insertion and query rates. However, the update of the band induces a high maintenance cost in terms of message overhead and data replication.

Dynamic prefix tree indexing structures create a dynamic trie by splitting overloaded leaf nodes according to data distribution. However, these strategies induce an index maintenance that consists in node coordination and data movement upon every index update. Examples of such structures are the Prefix Hash Tree [8] detailed in section 2 and LIGHT [9].

LIGHT is a dynamic tree similar to PHT. The main differences reside in the structure and the mapping of nodes onto the DHT ring. LIGHT only maps leaf nodes onto the DHT and does not maintain a doubly-linked list. This is possible because it defines a novel naming function that maps LIGHT nodes onto DHT nodes.

In order to perform index updates LIGHT defines a split operation named incremental leaf split. Upon every leaf split the local leaf node calculates the two next leaf labels by using the naming function, updates its label and moves the data only to one new leaf node following the prefix rule. When the index handles uniformly distributed data, LIGHT halves the data movement cost of PHT. However, LIGHT suffers from the same drawback as PHT when handling big data applications. As the number of keys stored onto each leaf node increases, the cost of index updates becomes unacceptable for many applications.

\section{Conclusion}

This paper presents FreeSplit, a new maintenance protocol for tree-based indexing systems. FreeSplit outperforms traditional existing solutions over DHTs like PHT and LIGHT in terms of time efficiency. This approach decreases the response time of queries, and thus significantly impacts the overall latency of the system. FreeSplit is particularly well suited for handling massive insertions of small objects in the context of Big Data applications. Knowledge of the object insertion rate for a given application allows adapting the maximum number of objects stored onto a leaf node so that the maintenance operations of FreeSplit are always performed in the best case, and therefore remain time efficient. It can easily be ported to various existing tree-based indexing systems, as well as over different structured peer-to-peer overlays. 
Future research directions include taking into account the presence of malicious nodes by using a more effective replication scheme combined with trust mechanisms. Another interesting problem is how to handle massive flows of big objects.

\section{References}

[1] S. Kaisler, F. Armour, J. Espinosa, and W. Money, "Big data: Issues and challenges moving forward," in 2013 46th Hawaii International Conference on System Sciences (HICSS), pp. 995-1004, 2013.

[2] M. Wigan and R. Clarke, "Big data's big unintended consequences," Computer, vol. 46, no. 6 , pp. $46-53,2013$.

[3] S. Chaudhuri, "How different is big data?," in 2012 IEEE 28th International Conference on Data Engineering (ICDE), pp. 5-5, 2012.

[4] L. Ferrari and M. Mamei, "Identifying and understanding urban sport areas using nokia sports tracker," Pervasive and Mobile Computing, vol. 9, no. 5, pp. 616-628, 2013.

[5] A. Clarke and R. Steele, "How personal fitness data can be re-used by smart cities," in Intelligent Sensors, Sensor Networks and Information Processing (ISSNIP), 2011 Seventh International Conference on, pp. 395-400, IEEE, 2011.

[6] M. Chen, S. Mao, and Y. Liu, "Big data: A survey," Mobile Networks and Applications, pp. 1-39, 2014.

[7] A. Zaslavsky, C. Perera, and D. Georgakopoulos, "Sensing as a service and big data," in Proceedings of the International Conference on Advances in Cloud Computing (ACC), Bangalore, India, July 2012.

[8] S. Ramabhadran, S. Ratnasamy, J. M. Hellerstein, and S. Shenker, "Brief announcement: Prefix hash tree," in Proceedings of the Twenty-third Annual ACM Symposium on Principles of Distributed Computing, PODC '04, p. 368-368, ACM, 2004.

[9] Y. Tang, S. Zhou, and J. Xu, "LIGHT: a query-efficient yet low-maintenance indexing scheme over DHTs," IEEE Transactions on Knowledge and Data Engineering, vol. 22, no. 1, pp. 59$75,2010$.

[10] C. Zheng, G. Shen, S. Li, and S. Shenker, "Distributed segment tree: Support of range query and cover query over dht," in In Electronic publications of the 5th International Workshop on Peer-to-Peer Systems (IPTPS'06, 2006.

[11] J. Gao and P. Steenkiste, "An adaptive protocol for efficient support of range queries in DHT-based systems," in Proceedings of the 12th IEEE International Conference on Network Protocols, 2004. ICNP 2004, pp. 239-250, 2004.

[12] A. Rowstron and P. Druschel, "Pastry: Scalable, decentralized object location, and routing for large-scale peer-to-peer systems," in Middleware 2001 (R. Guerraoui, ed.), no. 2218 in Lecture Notes in Computer Science, pp. 329-350, Springer Berlin Heidelberg, 2001.

[13] I. Stoica, R. Morris, D. Karger, M. F. Kaashoek, and H. Balakrishnan, "Chord: A scalable peer-to-peer lookup service for internet applications," in Proceedings of the 2001 Conference on Applications, Technologies, Architectures, and Protocols for Computer Communications, SIGCOMM '01, p. 149-160, ACM, 2001. 


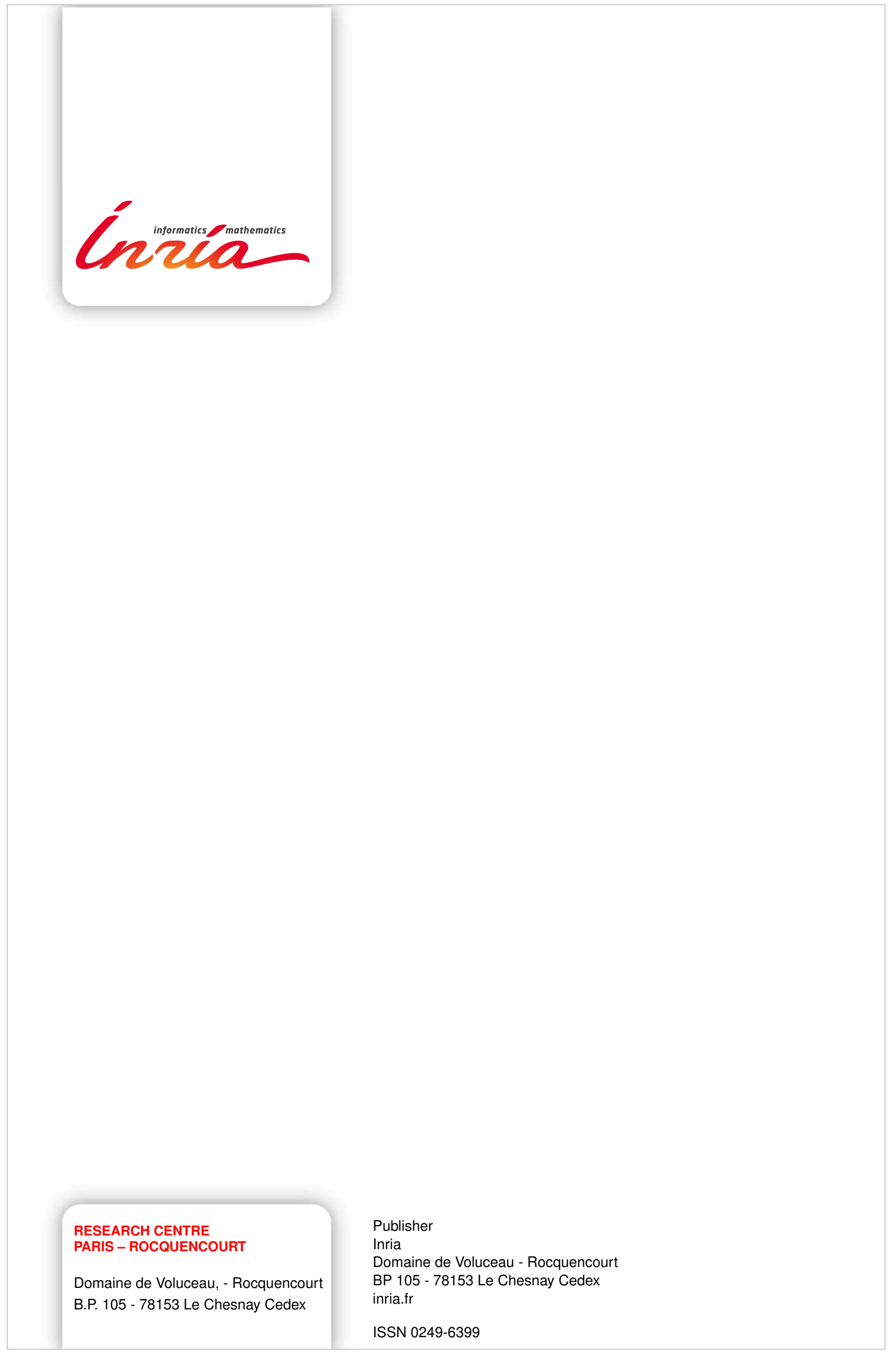

